\title{
COHOMOLOGICAL CHARACTERIZATIONS OF CHARACTER PSEUDO-AMENABLE BANACH ALGEBRAS
}

\author{
RASOUL NASR-ISFAHANI ${ }^{\bowtie}$ and MEHDI NEMATI
}

\author{
(Received 17 October 2010)
}

\begin{abstract}
For a Banach algebra $\mathcal{A}$ and a character $\phi$ on $\mathcal{A}$, we have recently introduced and studied the notion of $\phi$-pseudo-amenability of $\mathcal{A}$. Here, we give some characterizations of this notion in terms of derivations from $\mathcal{A}$ into various Banach $\mathcal{A}$-bimodules.
\end{abstract}

2010 Mathematics subject classification: primary 46H05; secondary 43A07.

Keywords and phrases: Banach algebra, pseudo-amenable, approximate diagonal, $\phi$-pseudo-amenable.

\section{Introduction}

Let $\mathcal{A}$ be a Banach algebra and let $\phi \in \sigma(\mathcal{A})$, the set of all nonzero homomorphisms from $\mathcal{A}$ onto $\mathbb{C}$. $\mathcal{A}$ is called $\phi$-amenable if there exists a bounded linear functional $F \in \mathcal{A}^{* *}$ satisfying $F(\phi)=1$ and $a \odot F=\phi(a) F$ for all $a \in \mathcal{A}$, where $\odot$ is the first Arens multiplication on the second dual $\mathcal{A}^{* *}$ of $\mathcal{A}$ defined by the equations

$$
(F \odot H)(f)=F(H f), \quad(H f)(a)=H(f a), \quad(f a)(b)=f(a b)
$$

for all $F, H \in \mathcal{A}^{* *}, f \in \mathcal{A}^{*}$, and $a, b \in \mathcal{A}$. This notion is introduced and studied by Kaniuth et al. [7]; see also [8]. The notion of character amenability is a generalization of left amenability of the class of $F$-algebras $\mathcal{L}$ studied in Lau [9] in 1983, known as Lau algebras; see Pier [12]. The class of Lau algebras includes the group algebra $L^{1}(G)$, and the measure algebra $M(G)$ of an amenable locally compact group $G$, as well as the Fourier and Fourier-Stieltjes algebras $A(G)$ and $B(G)$ of any $G$. It also includes the quantum group algebra $L^{1}(Q)$ when $Q$ is amenable. In this case, the character is taken to be the identity of the von Neumann algebra $\mathcal{L}^{*}$.

For a Banach $\mathcal{A}$-bimodule $X$, a derivation $D: \mathcal{A} \rightarrow X$ is a linear map such that

$$
D(a b)=a \cdot D(b)+D(a) \cdot b \quad(a, b \in \mathcal{A}) .
$$

For $x \in X$, define $\operatorname{ad}_{x}: \mathcal{A} \rightarrow X$ by $\operatorname{ad}_{x}(a)=a \cdot x-x \cdot a$ for all $a \in \mathcal{A}$. Then $\operatorname{ad}_{x}$ is a derivation; these are the inner derivations. Note that the dual space $X^{*}$ of a Banach

(C) 2011 Australian Mathematical Publishing Association Inc. 0004-9727/2011 \$16.00 
$\mathcal{A}$-bimodule $X$ becomes a Banach $\mathcal{A}$-bimodule with

$$
\langle a \cdot \xi, x\rangle=\langle\xi, x \cdot a\rangle, \quad\langle\xi \cdot a, x\rangle=\langle\xi, a \cdot x\rangle,
$$

for all $x \in X, \xi \in X^{*}$, and $a \in \mathcal{A}$. Following Hu et al. [6], for $\phi \in \sigma(\mathcal{A}) \cup\{0\}$, we denote by ${ }_{\phi} \mathcal{M}^{\mathcal{A}}$ the class of Banach $\mathcal{A}$-bimodules $X$ for which the left module action of $\mathcal{A}$ on $X$ is defined by

$$
a \cdot x=\phi(a) x \quad(a \in \mathcal{A}, x \in X) .
$$

The notion of $\phi$-amenability is characterized in several different ways; for example, it is equivalent to the notion that for each $X \in{ }_{\phi} \mathcal{M}^{\mathcal{A}}$, every continuous derivation $D$ : $\mathcal{A} \rightarrow X^{*}$ is inner [7]; moreover, it was shown in [6] that $\phi$-amenability is equivalent to the existence of a bounded (right) $\phi$-approximate diagonal; that is, a bounded net $\left(\mathbf{m}_{\alpha}\right)$ in the projective tensor product $\mathcal{A} \widehat{\otimes} \mathcal{A}$ such that

$$
\phi\left(\pi\left(\mathbf{m}_{\alpha}\right)\right) \rightarrow 1 \quad \text { and } \quad\left\|a \cdot \mathbf{m}_{\alpha}-\phi(a) \mathbf{m}_{\alpha}\right\| \rightarrow 0
$$

for all $a \in \mathcal{A}$, where $\pi$ denotes the product morphism from $\mathcal{A} \widehat{\otimes} \mathcal{A}$ into $\mathcal{A}$ given by $\pi(a \otimes b)=a b$ for all $a, b \in \mathcal{A}$; see also [1].

Moreover, Ghahramani and Zhang [5] introduced and studied the notion of pseudoamenability of $\mathcal{A}$, the existence of an approximate diagonal for $\mathcal{A}$; that is, a net $\left(\mathbf{m}_{\alpha}\right)$ in $\mathcal{A} \widehat{\otimes} \mathcal{A}$ such that

$$
\left\|\pi\left(\mathbf{m}_{\alpha}\right) a-a\right\| \rightarrow 0 \quad \text { and } \quad\left\|a \cdot \mathbf{m}_{\alpha}-\mathbf{m}_{\alpha} \cdot a\right\| \rightarrow 0
$$

for all $a \in \mathcal{A}$.

Recently, in [11] we introduced and studied a notion of amenability called $\phi$-pseudo-amenability. In this paper we characterize $\phi$-pseudo-amenability of $\mathcal{A}$ in terms of derivations from $\mathcal{A}$ into certain Banach $\mathcal{A}$-bimodules.

\section{The results}

Let $\mathcal{A}$ be a Banach algebra and let $X$ be a Banach $\mathcal{A}$-bimodule. A derivation $D$ : $\mathcal{A} \rightarrow X$ is approximately inner if there is a net $\left(x_{\alpha}\right) \subseteq X$ such that

$$
D(a)=\lim _{\alpha}\left(a \cdot x_{\alpha}-x_{\alpha} \cdot a\right) \quad(a \in \mathcal{A}) ;
$$

see, for example, $[2,4]$. The notion of $\phi$-approximate diagonal was introduced and studied by $\mathrm{Hu}$ et al. [6]. We commence this section with the main definition of the paper.

Definition 2.1. Let $\mathcal{A}$ be a Banach algebra and let $\phi \in \sigma(\mathcal{A})$. We say that $\mathcal{A}$ is $\phi$-pseudo-amenable if it has a (right) $\phi$-approximate diagonal (not necessarily bounded).

In our first result, we characterize $\phi$-pseudo-amenability of $\mathcal{A}$ in terms of derivations from $\mathcal{A}$ into duals of elements in ${ }_{\phi} \mathcal{M}^{\mathcal{A}}$.

Theorem 2.2. Let $\mathcal{A}$ be a Banach algebra and $\phi \in \sigma(\mathcal{A})$. Then the following statements are equivalent. 
(a) $\mathcal{A}$ is $\phi$-pseudo-amenable.

(b) For each $X \in{ }_{\phi} \mathcal{M}^{\mathcal{A}}$, any continuous derivation $D: \mathcal{A} \rightarrow X^{*}$ is approximately inner.

Proof. (a) $\Rightarrow$ (b). Suppose that $X \in{ }_{\phi} \mathcal{M}^{\mathcal{A}}$ and $D: \mathcal{A} \rightarrow X^{*}$ is a continuous derivation. Let $\left(\mathbf{m}_{\alpha}\right) \subseteq \mathcal{A} \widehat{\otimes} \mathcal{A}$ be a $\phi$-approximate diagonal for $\mathcal{A}$ and let $\Phi: \mathcal{A} \widehat{\otimes} \mathcal{A} \rightarrow$ $X^{*}$ be the bounded linear mapping specified by

$$
\Phi(a \otimes b)=D(a) \phi(b)
$$

for all $a, b \in \mathcal{A}$. Then $\|\Phi\| \leq\|D\|$ and

$$
\Phi\left(a \cdot \mathbf{m}_{\alpha}-\phi(a) \mathbf{m}_{\alpha}\right)=a \cdot \Phi\left(\mathbf{m}_{\alpha}\right)+\phi\left(\pi\left(\mathbf{m}_{\alpha}\right)\right) D(a)-\phi(a) \Phi\left(\mathbf{m}_{\alpha}\right)
$$

for all $a \in \mathcal{A}$. Let $\xi_{\alpha}:=-\Phi\left(\mathbf{m}_{\alpha}\right)$. Then for each $a \in \mathcal{A}$ we have

$$
\phi\left(\pi\left(\mathbf{m}_{\alpha}\right)\right) D(a)=a \cdot \xi_{\alpha}-\phi(a) \xi_{\alpha}+\Phi\left(a \cdot \mathbf{m}_{\alpha}-\phi(a) \mathbf{m}_{\alpha}\right) .
$$

On the other hand,

$$
\left\|\Phi\left(a \cdot \mathbf{m}_{\alpha}-\phi(a) \mathbf{m}_{\alpha}\right) \leq\right\| D\|\| a \cdot \mathbf{m}_{\alpha}-\phi(a) \mathbf{m}_{\alpha} \| \rightarrow 0
$$

for all $a \in \mathcal{A}$. It follows that

$$
D(a)=\lim _{\alpha}\left(a \cdot \xi_{\alpha}-\xi_{\alpha} \cdot a\right) \quad(a \in \mathcal{A}) .
$$

(b) $\Rightarrow$ (a). Suppose that for each $X \in{ }_{\phi} \mathcal{M}^{\mathcal{A}}$, every continuous derivation $D: \mathcal{A} \rightarrow$ $X^{*}$ is approximately inner. Consider the quotient Banach $\mathcal{A}$-bimodule $X=\mathcal{A}^{*} / \mathbb{C} \phi$. Let $F_{0} \in \mathcal{A}^{* *}$ be such that $F_{0}(\phi)=1$ and let $\operatorname{ad}_{F_{0}}: \mathcal{A} \rightarrow \mathcal{A}^{* *}$ be the inner derivation by $F_{0}$. Then the image of $\operatorname{ad}_{F_{0}}$ is a subset of $X^{*}$, and hence by our assumption, there exists a net

$$
\left(F_{\alpha}^{\prime}\right) \subseteq X^{*}=\left\{F \in \mathcal{A}^{* *}: F(\phi)=0\right\}
$$

such that

$$
\operatorname{ad}_{F_{0}}(a)=\lim _{\alpha} \operatorname{ad}_{F_{\alpha}^{\prime}}(a)
$$

for all $a \in \mathcal{A}$. Thus if we define $F_{\alpha}=F_{0}-F_{\alpha}^{\prime}$, then

$$
F_{\alpha}(\phi)=\left(F_{0}\right)(\phi)=1 \quad \text { and } \quad\left\|a \odot F_{\alpha}-\phi(a) F_{\alpha}\right\| \rightarrow 0
$$

for all $a \in \mathcal{A}$. We can use Goldstine's theorem to assume that $\left(F_{\alpha}\right)$ is in $\mathcal{A}$ when the convergence in the above equation is the weak convergence. Applying Mazur's theorem, we can get a new net $\left(a_{\alpha}\right) \subseteq \mathcal{A}$ such that

$$
\phi\left(a_{\alpha}\right)=1 \quad \text { and } \quad\left\|a a_{\alpha}-\phi(a) a_{\alpha}\right\| \rightarrow 0 .
$$

Now, choose $a_{0} \in \mathcal{A}$ with $\phi\left(a_{0}\right)=1$ and put

$$
\mathbf{m}_{\alpha}:=a_{\alpha} \otimes a_{0} .
$$

It is easy to check that $\left(\mathbf{m}_{\alpha}\right)$ is a $\phi$-approximate diagonal for $\mathcal{A}$. Thus $\mathcal{A}$ is $\phi$-pseudoamenable. 
Definition 2.3. Let $\mathcal{A}$ be a Banach algebra. We say that $\mathcal{A}$ is 0-pseudo-amenable if it has a right approximate identity.

TheOREM 2.4. Let $\mathcal{A}$ be a Banach algebra. Then $\mathcal{A}$ is 0-pseudo-amenable if and only if every continuous derivation $D: \mathcal{A} \rightarrow X^{*}$ is approximately inner for all $X \in{ }_{0} \mathcal{M}^{\mathcal{A}}$.

Proof. The 'if' part follows from [4, Proof of Lemma 2.2]. To prove the converse, suppose that $\left(a_{\alpha}\right) \subseteq \mathcal{A}$ is a right approximate identity for $\mathcal{A}$ and suppose that $D$ : $\mathcal{A} \rightarrow X^{*}$ is a continuous derivation, where $X \in{ }_{0} \mathcal{M}^{\mathcal{A}}$. We set

$$
\xi_{\alpha}:=D\left(a_{\alpha}\right)
$$

for all $\alpha$. Therefore for each $a \in \mathcal{A}$ we have

$$
\begin{aligned}
D(a) & =\lim _{\alpha} D\left(a a_{\alpha}\right)=\lim _{\alpha} D(a) \cdot a_{\alpha}+a \cdot D\left(a_{\alpha}\right) \\
& =\lim _{\alpha} a \cdot D\left(a_{\alpha}\right)=\lim _{\alpha} a \cdot \xi_{\alpha} \\
& =\lim _{\alpha} a \cdot \xi_{\alpha}-\xi_{\alpha} \cdot a .
\end{aligned}
$$

Thus $D$ is approximately inner.

A Banach algebra $\mathcal{A}$ is approximately amenable if every continuous derivation from $\mathcal{A}$ into the dual $\mathcal{A}$-bimodule $X^{*}$ is approximately inner for all Banach $\mathcal{A}$-bimodules $X$; this notion was introduced and studied by Ghahramani and Loy [4]. Moreover, the notion of character amenability was introduced and studied by Monfared [10]; he called a Banach algebra $\mathcal{A}$ character amenable if it has a bounded right approximate identity and is $\phi$-amenable for all nonzero characters $\phi$ on $\mathcal{A}$. We are thus led to the following definition.

Definition 2.5. A Banach algebra $\mathcal{A}$ is called character pseudo-amenable if it is $\phi$-pseudo-amenable for all $\phi \in \sigma(\mathcal{A}) \cup\{0\}$.

As an application of Theorems 2.2 and 2.4 we have the following result.

Corollary 2.6. Let $\mathcal{A}$ be an approximately amenable Banach algebra. Then $\mathcal{A}$ is character pseudo-amenable.

The following example shows that the converse of the above corollary is not true.

ExAmple 2.7. (a) Let $1 \leq p<\infty$ and let $S$ be an infinite set. Consider the Banach algebra $\ell^{p}(S)$ of all complex-valued functions $f:=\sum_{s \in S} f(s) \delta_{s}$ with

$$
\|f\|^{p}:=\sum_{s \in S}|f(s)|^{p}<\infty
$$

endowed with the pointwise multiplication, where $\delta_{s}$ is the characteristic function of $\{s\}$. First note that

$$
\sigma\left(\ell^{p}(S)\right)=\left\{\phi_{s}: s \in S\right\}
$$


where $\phi_{s}(f)=f(s)$ for all $f \in \ell^{p}(S)$. Now, suppose that $X \in{ }_{\phi_{s}} \mathcal{M}^{\ell^{p}(S)}$ and $D$ : $\ell^{p}(S) \rightarrow X^{*}$ is a continuous derivation. Then

$$
D\left(f \delta_{s}\right)=f \cdot D\left(\delta_{s}\right)+\phi_{s}\left(\delta_{s}\right) D(f)
$$

for all $f \in \ell^{p}(S)$ and $s \in S$. Since $f \delta_{s}=f(s) \delta_{s}=\phi_{s}(f) \delta_{s}$ and $\phi_{s}\left(\delta_{s}\right)=1$, it follows that

$$
D(f)=\phi_{s}(f) D\left(\delta_{s}\right)-f \cdot D\left(\delta_{s}\right) .
$$

Thus $D=\operatorname{ad}_{-D\left(\delta_{s}\right)}$ and hence $\ell^{p}(S)$ is $\phi_{s}$-pseudo-amenable for all $s \in S$ by Theorem 2.2. Moreover, $\ell^{p}(S)$ has an approximate identity, and consequently it is character pseudo-amenable. On the other hand, it was shown in [3] that the Banach algebra $\ell^{p}(S)$ is not approximately amenable.

(b) Let $G$ be an infinite compact abelian group. Then since $G$ is compact and abelian, the Feichtinger algebra is

$$
S_{0}(G)=\left\{f=\sum_{\rho \in \widehat{G}} c_{\rho} \delta_{\rho}:\|f\|=\sum\left|c_{\rho}\right|<\infty\right\},
$$

where $\widehat{G}$ is the dual group of $G$. Hence,

$$
S_{0}(G) \cong \ell^{1}(\widehat{G})
$$

where $\ell^{1}(\widehat{G})$ is equipped with the pointwise product. Thus $S_{0}(G)$ is character pseudoamenable, but not approximately amenable.

Let $\mathcal{M}_{\phi}^{\mathcal{A}}$ denote the class of Banach $\mathcal{A}$-bimodules $X$ for which the right module action of $\mathcal{A}$ on $X$ is defined by $x \cdot a=\phi(a) x(a \in \mathcal{A}, x \in X)$. We have the following analogue of a result in [6] on $\phi$-amenable Banach algebras.

Proposition 2.8. Let $\mathcal{A}$ be a Banach algebra and $\phi \in \sigma(\mathcal{A}) \cup\{0\}$. Then the following statements are equivalent.

(a) $\mathcal{A}$ is $\phi$-pseudo-amenable.

(b) For every $X \in \mathcal{M}_{\phi}^{\mathcal{A}}$, any continuous derivation $D: \mathcal{A} \rightarrow X^{* *}$ is approximately inner.

(c) For every $X \in \mathcal{M}_{\phi}^{\mathcal{A}}$, any continuous derivation $D: \mathcal{A} \rightarrow X$ is approximately inner.

Proof. Clearly, (a) implies (b) and (c) implies (a) by Theorem 2.2. Thus it suffices to show that (b) implies (c). Suppose that $X \in \mathcal{M}_{\phi}^{\mathcal{A}}$ and $D: \mathcal{A} \rightarrow X$ is a continuous derivation. Then the map $\Delta:\left.D^{* *}\right|_{\mathcal{A}} \rightarrow X^{* *}$ is a derivation. By assumption, there exists a net $\left(\Xi_{\alpha}\right) \subseteq X^{* *}$ such that

$$
\Delta(a)=D(a)=\lim _{\alpha}\left(a \cdot \Xi_{\alpha}-\Xi_{\alpha} \cdot a\right) .
$$

Then by Goldstine's theorem, we can assume that $\Xi_{\alpha} \in X$ when the above limit converges in the weak topology of $X$. Applying Mazur's theorem, we can get a new 
net $\left(x_{\alpha}\right) \subseteq X$ such that

$$
D(a)=\lim _{\alpha}\left(a \cdot x_{\alpha}-x_{\alpha} \cdot a\right)
$$

for all $a \in \mathcal{A}$, and this completes the proof.

Corollary 2.9. Let $\mathcal{A}$ be a finite-dimensional Banach algebra and $\phi \in \sigma(\mathcal{A}) \cup\{0\}$. Then $\mathcal{A}$ is $\phi$-pseudo-amenable if and only if $\mathcal{A}$ is $\phi$-amenable.

Proof. Suppose that $D: \mathcal{A} \rightarrow X^{*}$ is a continuous derivation for some $X \in{ }_{\phi} \mathcal{M}^{\mathcal{A}}$. Let $E$ be the subspace of $X^{*}$ generated by $D(\mathcal{A})+\mathcal{A} \cdot D(\mathcal{A})$. Thus $E$ is a finitedimensional submodule of $X^{*}$ and $E \in \mathcal{M}_{\phi}^{\mathcal{A}}$. Using the fact that

$$
\mathcal{B}^{1}(\mathcal{A}, E)=\left\{\operatorname{ad}_{\xi}: \xi \in E\right\}
$$

is a finite-dimensional space, we conclude that it is a closed subspace of $\mathcal{L}(\mathcal{A}, E)$, the set of bounded linear mappings from $\mathcal{A}$ into $E$, in the strong operator topology. Define $D_{E}: \mathcal{A} \rightarrow E$ by $D_{E}(a)=D(a)$ for all $a \in \mathcal{A}$. Since $\mathcal{A}$ is $\phi$-pseudo-amenable and $E \in \mathcal{M}_{\phi}^{\mathcal{A}}$, there exists a net $\left(\xi_{\alpha}\right)$ in $E$ such that for each $a \in \mathcal{A}$,

$$
D_{E}(a)=\lim _{\alpha} \operatorname{ad}_{\xi_{\alpha}}(a)
$$

by Proposition 2.8. It follows that $D_{E} \in \mathcal{B}^{1}(\mathcal{A}, E)$, and hence there exists $\xi \in E \subseteq X^{*}$ such that $D_{E}=\operatorname{ad}_{\xi}$. Therefore $D$ is inner.

Let $\mathcal{A}$ be a Banach algebra and $\phi \in \sigma(\mathcal{A}) \cup\{0\}$. Then we can consider $\operatorname{ker}(\phi) \in$ $\mathcal{M}_{\phi}^{\mathcal{A}}$ with the right action

$$
b \cdot a=\phi(a) b \quad(a \in \mathcal{A}, b \in \operatorname{ker}(\phi)),
$$

and the left action to be the natural one. In the following result we consider $\operatorname{ker}(\phi)$ as an $\mathcal{A}$-bimodule with these actions.

Corollary 2.10. Let $\mathcal{A}$ be a Banach algebra and $\phi \in \sigma(\mathcal{A}) \cup\{0\}$. Then the following statements are equivalent.

(a) $\mathcal{A}$ is $\phi$-pseudo-amenable.

(b) Any continuous derivation $D: \mathcal{A} \rightarrow(\operatorname{ker}(\phi))^{* *}$ is approximately inner.

(c) Any continuous derivation $D: \mathcal{A} \rightarrow \operatorname{ker}(\phi)$ is approximately inner.

Proof. Clearly, (a) implies (b) and (b) implies (c) by Proposition 2.8. It suffices to show that (c) implies (a). To that end first suppose that $\phi \in \sigma(\mathcal{A})$ and choose any $b \in \mathcal{A}$ with $\phi(b)=1$. Then

$$
D(a)=a b-\phi(a) b \quad(a \in \mathcal{A})
$$

defines a continuous derivation from $\mathcal{A}$ into $\operatorname{ker}(\phi)$. Thus $D$ is approximately inner by (c), and so there is a net $\left(b_{\alpha}\right) \subseteq \operatorname{ker}(\phi)$ such that

$$
D(a)=\lim _{\alpha}\left(a b_{\alpha}-\phi(a) b_{\alpha}\right)
$$


for all $a \in \mathcal{A}$. If we set $a_{\alpha}:=b-b_{\alpha}$ for all $\alpha$, then we have $\phi\left(a_{\alpha}\right)=1$ and for each $a \in \mathcal{A}$,

$$
a a_{\alpha}-\phi(a) a_{\alpha} \rightarrow 0
$$

Trivially,

$$
\mathbf{m}_{\alpha}:=a_{\alpha} \otimes a_{0}
$$

is a $\phi$-approximate diagonal for $\mathcal{A}$, where $a_{0} \in \mathcal{A}$ with $\phi\left(a_{0}\right)=1$. Therefore $\mathcal{A}$ is $\phi$-pseudo-amenable. For the case $\phi=0$, the proof is similar to the proof of Theorem 2.4.

Let $\Theta: \mathcal{A} \rightarrow \mathcal{B}$ be a Banach algebra homomorphism and $\phi \in \sigma(\mathcal{A}) \cup\{0\}$. Then $\mathcal{B}$ is a Banach $\mathcal{A}$-bimodule by the module actions

$$
a \cdot b=\Theta(a) b, \quad b \cdot a=\phi(a) b \quad(a \in \mathcal{A}, b \in \mathcal{B}) .
$$

We denote by $\mathcal{B}_{\phi}^{\Theta}$ the above $\mathcal{A}$-bimodule in $\mathcal{M}_{\phi}^{\mathcal{A}}$.

THEOREM 2.11. Let $\mathcal{A}$ be a Banach algebra and $\phi \in \sigma(\mathcal{A}) \cup\{0\}$. Then the following statements are equivalent.

(a) $\mathcal{A}$ is $\phi$-pseudo-amenable.

(b) For every Banach algebra $\mathcal{B}$ and every homomorphism $\Theta: \mathcal{A} \rightarrow \mathcal{B}$, any continuous derivation $D: \mathcal{A} \rightarrow \mathcal{B}_{\phi}^{\Theta}$ is approximately inner.

(c) For every Banach algebra $\mathcal{B}$ and every injective homomorphism $\Theta: \mathcal{A} \rightarrow \mathcal{B}$, any continuous derivation $D: \mathcal{A} \rightarrow \mathcal{B}_{\phi}^{\Theta}$ is approximately inner.

Proof. The implications (a) $\Rightarrow$ (b) $\Rightarrow$ (c) are trivial. We show that (c) $\Rightarrow$ (a) holds. Suppose that (c) holds and let $X \in \mathcal{M}_{\phi}^{\mathcal{A}}$ and $D: \mathcal{A} \rightarrow X$ be a continuous derivation. Consider the module extension Banach algebra $X \oplus_{1} \mathcal{A}$; that is, the space $X \oplus \mathcal{A}$ endowed with the norm

$$
\|(x, a)\|=\|x\|+\|a\| \quad(a \in \mathcal{A}, x \in X)
$$

and the product

$$
\left(x_{1}, a_{1}\right)\left(x_{2}, a_{2}\right)=\left(x_{1} \cdot a_{2}+a_{1} \cdot x_{2}, a_{1} a_{2}\right)
$$

for all $a_{1}, a_{2} \in \mathcal{A}$ and $x_{1}, x_{2} \in X$. Obviously the map $\Theta: \mathcal{A} \rightarrow X \oplus_{1} \mathcal{A}$ defined by

$$
\Theta(a)=(0, a) \quad(a \in \mathcal{A})
$$

is an injective Banach algebra homomorphism. Now, if we define $D_{1}: \mathcal{A} \rightarrow X \oplus_{1} \mathcal{A}$ by

$$
D_{1}(a)=(D(a), 0) \quad(a \in \mathcal{A}),
$$

then for each $a, b \in \mathcal{A}$ we have

$$
\begin{aligned}
D_{1}(a b)=(D(a b), 0) & =(\phi(b) D(a)+a \cdot D(b), 0) \\
& =\phi(b)(D(a), 0)+(0, a)(D(b), 0) \\
& =\phi(b) D_{1}(a)+\Theta(a) D_{1}(b) .
\end{aligned}
$$


Thus, $D_{1}$ is a derivation from $\mathcal{A}$ into $\left(X \oplus_{1} \mathcal{A}\right)_{\phi}^{\Theta}$, and so $D_{1}$ is approximately inner by assumption. That is, there exist nets $\left(a_{\alpha}\right) \subseteq \mathcal{A}$ and $\left(x_{\alpha}\right) \subseteq X$ such that

$$
D_{1}(a)=\lim _{\alpha} \operatorname{ad}_{\left(x_{\alpha}, a_{\alpha}\right)}(a)
$$

for all $a \in \mathcal{A}$. Thus, for each $a \in \mathcal{A}$ we have

$$
\begin{aligned}
(D(a), 0)=D_{1}(a) & =\lim _{\alpha} \operatorname{ad}_{\left(x_{\alpha}, a_{\alpha}\right)}(a) \\
& =\lim _{\alpha}\left(\Theta(a)\left(x_{\alpha}, a_{\alpha}\right)-\phi(a)\left(x_{\alpha}, a_{\alpha}\right)\right) \\
& =\lim _{\alpha}\left((0, a)\left(x_{\alpha}, a_{\alpha}\right)-\phi(a)\left(x_{\alpha}, a_{\alpha}\right)\right) \\
& =\lim _{\alpha}\left(a x_{\alpha}-\phi(a) x_{\alpha}, a a_{\alpha}-\phi(a) a_{\alpha}\right) .
\end{aligned}
$$

Therefore, $D(a)=\lim _{\alpha} \operatorname{ad}_{x_{\alpha}}(a)$. So, for each $X \in \mathcal{M}_{\phi}^{\mathcal{A}}$, any continuous derivation $D: \mathcal{A} \rightarrow X$ is approximately inner; this is equivalent to $\phi$-pseudo-amenability by Proposition 2.8.

We end this work with the following description of $\phi$-amenability which is of interest in its own right.

Theorem 2.12. Let $\mathcal{A}$ be a Banach algebra and $\phi \in \sigma(\mathcal{A})$. Then $\mathcal{A}$ is $\phi$-pseudoamenable if and only if there exists a net $\left(\mathbf{n}_{\alpha}\right) \subseteq \mathcal{A} \widehat{\otimes} \mathcal{A}$ such that $\phi\left(\pi\left(\mathbf{n}_{\alpha}\right)\right) \rightarrow 1$ and $\left\|a \cdot \mathbf{n}_{\alpha}\right\| \rightarrow 0$ for all $a \in \operatorname{ker}(\phi)$.

PROOF. The 'only if' part follows from the definition of $\phi$-pseudo-amenability and $\phi$-approximate diagonal. Now, assume that there exists a net $\left(\mathbf{n}_{\alpha}\right) \subseteq \mathcal{A} \widehat{\otimes} \mathcal{A}$ such that

$$
\phi\left(\pi\left(\mathbf{n}_{\alpha}\right)\right) \rightarrow 1 \quad \text { and } \quad\left\|a \cdot \mathbf{n}_{\alpha}\right\| \rightarrow 0
$$

for all $a \in \operatorname{ker}(\phi)$. Choose $a_{0} \in \mathcal{A}$ with $\phi\left(a_{0}\right)=1$. Then $a a_{0}-\phi(a) a_{0} \in \operatorname{ker}(\phi)$ for all $a \in \mathcal{A}$. Set

$$
\mathbf{m}_{\alpha}:=a_{0} \cdot \mathbf{n}_{\alpha}
$$

for all $\alpha$. Thus $\phi\left(\pi\left(\mathbf{m}_{\alpha}\right)\right)=\phi\left(a_{0} \pi\left(\mathbf{n}_{\alpha}\right)\right) \rightarrow 1$ and, for each $a \in \mathcal{A}$,

$$
\left\|a \cdot \mathbf{m}_{\alpha}-\phi(a) \mathbf{m}_{\alpha}\right\|=\left\|\left(a a_{0}-\phi(a) a_{0}\right) \cdot \mathbf{n}_{\alpha}\right\| \rightarrow 0 .
$$

This shows that $\left(\mathbf{m}_{\alpha}\right)$ is a $\phi$-approximate diagonal for $\mathcal{A}$.

\section{References}

[1] M. Alaghmandan, R. Nasr-Isfahani and M. Nemati, 'Character amenability and contractibility of abstract Segal algebras', Bull. Aust. Math. Soc. 82 (2010), 274-281.

[2] P. Bharucha, 'Approximate weak amenability of $I_{0}(\operatorname{SL}(2, \mathbb{R}))$ ', Bull. Aust. Math. Soc. 82 (2010), 18-21.

[3] H. G. Dales, R. J. Loy and Y. Zhang, 'Approximate amenability for Banach sequence algebras', Studia Math. 177 (2006), 81-96. 
[4] F. Ghahramani and R. J. Loy, 'Generalized notations of amenability', J. Funct. Anal. 208 (2004), 229-260.

[5] F. Ghahramani and Y. Zhang, 'Pseudo-amenable and pseudo-contractible Banach algebras', Math. Proc. Cambridge Philos. Soc. 142 (2007), 111-123.

[6] Z. Hu, M. S. Monfared and T. Traynor, 'On character amenable Banach algebras', Studia Math. 193 (2009), 53-78.

[7] E. Kaniuth, A. T. Lau and J. Pym, 'On $\phi$-amenability of Banach algebras', Math. Proc. Cambridge Philos. Soc. 144 (2008), 85-96.

[8] E. Kaniuth, A. T. Lau and J. Pym, 'On character amenability of Banach algebras', J. Math. Anal. Appl. 344 (2008), 942-955.

[9] A. T. Lau, 'Analysis on a class of Banach algebras with applications to harmonic analysis on locally compact groups and semigroups', Fund. Math. 118 (1983), 161-175.

[10] M. S. Monfared, 'Character amenability of Banach algebras', Math. Proc. Cambridge Philos. Soc. 144 (2008), 697-706.

[11] R. Nasr-Isfahani and M. Nemati, 'Character pseudo-amenability of Banach algebras', Preprint.

[12] J. P. Pier, Amenable Banach Algebras, Pitman Research Notes in Mathematics Series, 172 (Longman Scientific and Technical, Harlow, 1988).

RASOUL NASR-ISFAHANI, Department of Mathematical Sciences, Isfahan University of Technology, Isfahan 84156-83111, Iran

e-mail: isfahani@cc.iut.ac.ir

MEHDI NEMATI, Department of Mathematical Sciences, Isfahan University of Technology, Isfahan 84156-83111, Iran

e-mail: m.nemati@math.iut.ac.ir 